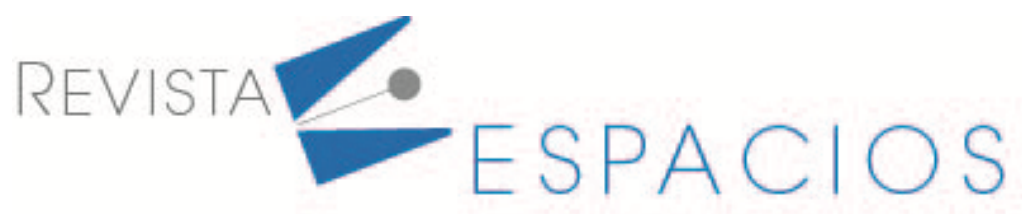

\title{
Las TICs como recurso para optimizar los procesos de enseñanza aprendizaje en la Facultad de Ciencias Matemáticas de la Universidad de Guayaquil (Ecuador)
}

Tics as a resource to optimize learning teaching processes at the Faculty of Mathematical Sciences of the University of Guayaquil

\author{
VILLALVA, Edgar E. ${ }^{1}$ \\ MOLINA, Rosa E. ${ }^{2}$ \\ PALACIOS, Francisco G. ${ }^{3}$ \\ VILLALVA, Marco A. ${ }^{4}$

\begin{abstract}
Resumen
En el estudio se analizó el conocimiento sobre TICs que tienen los estudiantes de la Facultad de Ciencias Matemáticas y Físicas de la Universidad de Guayaquil. La fundamentación teórica se basó en temáticas relacionadas con tics, aprendizaje y estudiantes. La investigación fue de tipo cuantitativo con enfoque descriptivo. Para probar los supuestos planteados se realizó encuestas aplicadas a 368 personas escogidas mediante muestreo estratificado de entre 4556 estudiantes legalmente matriculados en las 3 carreras que tuvo la Facultad durante el ciclo II 2019, lo cual permitió sacar conclusiones sobre varios aspectos a mejorar en los procesos de aprendizaje en la UG.
\end{abstract}

Palabras clave: TICS, Estudiantes, Proceso enseñanza aprendizaje

\begin{abstract}
The study analyzed the knowledge about TIC that students of the Faculty of Mathematical and Physical Sciences of the University of Guayaquil have. The theoretical foundation was based on topics related to tics, learning and students. The research was quantitative type with a descriptive approach. To test the assumptions raised, surveys were carried out on 368 persons chosen by stratified sampling of among 4556 students legally enrolled in the 3 careers that the Faculty had during the second cycle 2019, which allowed to take conclusions on various aspects to be improved in the learning processes in the UG.

key words: TICS, Students, Learning teaching process
\end{abstract}

\section{Introducción}

Actualmente las Tecnologías de la Información y las Comunicaciones (TIC) constituyen un componente esencial para el desarrollo de la Sociedad y es innegable que ha generado un cambio profundo en el desarrollo económico, social y cultural, permitiendo replantear el rol de la educación en todas sus etapas. A pesar de que las

\footnotetext{
${ }^{1}$ Docente, Facultad de Ciencias Administrativas, Universidad de Guayaquil, email: edgar.villalvac@ug.edu.ec

2 Docente, Facultad de Ciencias Matemáticas y Físicas, Universidad de Guayaquil, email: rosa.molinai@ug.edu.ec

${ }^{3}$ Docente, Facultad de Ciencias Matemáticas y Físicas, Universidad de Guayaquil, email: francisco.palacioso@ug.edu.ec

${ }^{4}$ Docente, Facultad de Ciencias Matemáticas y Físicas, Universidad de Guayaquil, email: marco.villalvac@ug.edu.ec
} 
herramientas de la Web 2.0 no fueron diseñadas con fines educativos, hoy son un recurso muy importante para abordar los cambios y nuevos paradigmas que se dan en los procesos de enseñanza-aprendizaje, Maloney, E. J. (2007), pero, es necesario analizar que las TICs así como tienen fortalezas también presentan debilidades que deben ser superadas para que sea optimizada su utilización.

Estas tecnologías innovan día a día, proporcionando en el ámbito educacional nuevos ambientes de aprendizaje, dejando de lado los métodos convencionales de enseñanza, y proponiendo un modelo educativo combinado que hace la fusión de los componentes presenciales con los no presenciales en donde las plataformas de aprendizaje elearning ayudan en la formación digital de profesionales, Acosta, J. Z., Vallejo, M., \& Uribe, I. (2017), facilitando los procesos pues hará que la enseñanza sea más manejable ya que se puede interactuar con los estudiantes desde fuera del aula, y en cualquier lugar y momento, Rodríguez Izquierdo, R. (2010).

Las tecnologías de la información y la comunicación están cambiando de forma radical nuestra forma de interrelacionarnos en cualquier ámbito de la vida. Como resultado lógico la educación no ha permanecido al margen a esta realidad, ya que el proceso educativo en sus diferentes variantes se apoya en el uso de diferentes TICS, adicionalmente en algunos casos se basa en el uso de Internet promoviendo un aprendizaje colaborativo, abierto y flexible, Castellano M. (2013). Es innegable que nos encontramos inmersos en una cultura digital. Por lo tanto, debemos aprovechar las bondades de este recurso, para potenciar el aprendizaje en los estudiantes.

El sistema educativo requiere transformar modelos, modos, medios y métodos educativos y de enseñanza aprendizaje con el propósito de utilizar adecuadamente las TIC. Debe implementar prácticas innovadoras, que permitan a la población estudiantil acceder a la sociedad del conocimiento, es urgente potenciar habilidades en estudiantes y en docentes. Es imperioso incorporar una educación digital, audiovisual y multimedia; para que este objetivo se cumpla es necesario el desarrollo de destrezas para localizar, organizar, entender, evaluar y analizar información de forma crítica y selectiva.

Los métodos y herramientas de enseñanza - aprendizaje que han sido utilizadas en los últimos 10 años en las diferentes universidades del Ecuador no han sido eficaces, más que todo en las carreras ligadas con la tecnología ya que al utilizar metodologías antiguas no hay la oportunidad de retroalimentarse con los avances respecto a los temas cubiertos en el aula, imposibilitando a adquirir nuevos conocimientos puesto que lo impartido en el salón de clases se quedan únicamente allí, sin la posibilidad de ligar lo aprendido con herramientas donde se involucre uso de las TICS. Las metodologías usadas por los docentes en las Instituciones de Educación Superior no tienen un gran enfoque andragógico es decir que no están orientadas a la forma como aprenden los adultos explotando su creatividad, imaginación y reflexión lo cual junto con las TICS podría muy bien ser usado para potenciar el aprendizaje en los estudiantes, Villalva E. (2018).

El docente debe ser consciente de que el mundo educativo demanda lo que el entorno en el que tiene lugar la enseñanza y la formación necesita, el cual se vuelve dinámico y complejo debido precisamente al desarrollo de las nuevas tecnologías. Por lo tanto, se hace necesario que el formador adquiera los conocimientos suficientes e imprescindibles en el uso de las nuevas tecnologías, así como una actitud positiva hacia éstas. De acuerdo con Hernández R. (2017), en el ámbito de la formación es "un reto integrar las nuevas tecnologías, de modo que mejore el rendimiento y la calidad de la práctica educativa y responder eficazmente a las demandas formativas". El profesor debe ser el motor para realizar los cambios requeridos en la educación pero como lo reseña Bawden, "los miembros de la comunidad académica no se sienten demasiado cómodos con la idea de ser agentes de cambio y desarrollo", Bawden R. (2009).

El maestro como facilitador educativo a parte del conocimiento sobre la materia que imparte, también debe manejar las TIC y ponerlas al servicio de los estudiantes como herramientas de apoyo para el aprendizaje, García B., Loredo J., Carranza G. (2008), debe convertirse en un asesor integral tanto científico como metodológico, 
siendo un guía a lo largo de todo el proceso, motivando y comprometiendo al alumno para que aprenda, así como fomentar en él una cultura de generación de sus propios aprendizajes. Las TIC aumentan las capacidades intelectuales, las potencian y permiten abrir posibilidades y nuevas formas de adquirir conocimiento, Viñals $A$, Cuenca J. (2015).

Es de vital importancia implementar acciones para ayudar a los docentes universitarios para que así puedan desarrollar diversas técnicas y estrategias para el uso general de las tecnologías. La Universidad de Guayaquil si cuenta con algunos recursos como por ejemplo la plataforma en la nube de Microsoft, Office 365, la cual no se aprovecha a un $100 \%$, esta herramienta es entregada de forma gratuita a los estudiantes, pero por falta de capacitación no la utilizan. Por tanto, existe una necesidad de que tanto los docentes como los estudiantes estén correctamente preparados y capacitados en el manejo de esta herramienta tecnológica, además de otras herramientas que se pueden anexar al Office 365 cuyo uso debe ser innato para estudiante de carrera ligada con la tecnología. Las Instituciones de Educación Superior deben "transformar" y amoldar su visión de formadores integrando a los procesos formativos la investigación, las nuevas herramientas de información y comunicación ajustándolas a las nuevas realidades que las sociedades del conocimiento están exigiendo, Flórez M., Aguilar A. (2017).

\section{Metodología}

El presente estudio tiene un enfoque cuantitativo, considerando que se recopila, mide, y tabula información cuántica, sin embargo, para el análisis e interpretación de los resultados, se utilizó técnicas cualitativas, la investigación es de carácter descriptivo, puesto que, se describen los hechos o fenómenos tal y cual se muestran; es documental y de campo pues se reviso información bibliográfica respecto a la temática tratada y se realizó una encuesta en el lugar donde se desenvuelven todos los involucrados, por el tiempo en que se realizo el estudio se la puede considerar de tipo transversal.

Para determinar los alcances del mismo se involucró a los estudiantes de la Facultad de Ciencias Matemáticas y Físicas de la Universidad de Guayaquil legalmente matriculados en el segundo ciclo del año 2019, información que se resume en la siguiente tabla.

Tabla 1

Población

\begin{tabular}{|clcc|}
\hline № & Detalle & Cantida & $\%$ \\
\hline 1 & Estudiantes de Ing. En Sistemas & 1229 & 27 \\
\hline 2 & Estudiantes de Ing. en Software & 579 & 13 \\
\hline 3 & $\begin{array}{l}\text { Estudiantes de Ing. en } \\
\text { Networking }\end{array}$ & 1038 & 23 \\
\hline 4 & $\begin{array}{l}\text { Estudiantes de Ing. en } \\
\text { Tecnolog. Información }\end{array}$ & 426 & 9 \\
\hline 5 & Estudiantes de Ing. Civil & 1284 & 28 \\
\hline \hline & Totales & $\mathbf{4 5 5 6}$ & $\mathbf{1 0 0}$ \\
\hline
\end{tabular}

El análisis se realizó tomando una muestra aleatoria de 368 estudiantes seleccionados de manera estratificada entre primero a octavo semestre de todas las carreras que tiene la facultad de Ciencias Matemáticas y Físicas, para obtener la muestra se utilizó una varianza media de 0,25 lo cual es sugerido en estudios relacionados al ámbito educacional, un margen de error del $5 \%$ y el valor de la constante de corrección igual a 2. 
Con el propósito de dar respuestas concretas a los objetivos planteados en la investigación, se utilizó la técnica de la encuesta usando un cuestionario el cual fue respondido por los estudiantes que formaron parte de la muestra lo cual fue realizado tanto en forma impresa como de manera digital usando google forms.

Para analizar los resultados de los cuestionarios se utilizó el software estadístico SPSS versión 23 para Windows; las preguntas que se incluyó en el instrumento fueron evaluadas obteniéndose las respectivas frecuencias absolutas y acumuladas.

La información de las fuentes primarias (docentes y alumnos) se las obtuvo mediante observación y encuestas, y para las fuentes secundarias se revisó artículos y ensayos sobre el uso de las TICS en el ámbito universitario.

\section{Resultados}

Los resultados que se obtuvieron de las encuestas aplicadas a los investigados fueron los siguientes:

El $83,68 \%$ de los estudiantes tienen una edad que oscila entre 17 y 25 años lo cual es bueno porque facilitaría el aprendizaje de cualquier herramienta informática que se les presente, adicionalmente se conoció que el $54.6 \%$ de los encuestados no trabajan y el $45.4 \%$ si lo hacen, lo cual es preocupante pues en este tipo de carreras tecnológicas se debe propender a tener estudiantes dedicados exclusivamente a sus estudios.

El 84.16\% indicó que si conoce lo que son las TICs y cuando se les pregunto qué tanto conocían mencionaron que sus conocimientos oscilan entre un 50 a $60 \%$. Adicionalmente de aquellas personas que respondieron afirmativamente a la pregunta, pues el $75.41 \%$ indicó que los docentes de las diferentes carreras de la facultad utilizan alguna herramienta TIC al momento de impartir sus clases a pesar que un 53\% indicó que los recursos tecnológicos ofrecidos por la institución tanto en hardware como en software para que los docentes impartan sus clases son pésimos o regulares y un $34.8 \%$ considera que son medianamente buenos lo cual debe mejorar ostensiblemente si se quiere ofertar una educación de calidad a los estudiantes de las diferentes carreras que tiene la facultad.

Al preguntar a los encuestados que herramientas TICs conocen y han utilizado como soporte para el aprendizaje de las diferentes asignaturas todos indicaron que en algún momento habían utilizado la plataforma edmodo, 357 han usado las herramientas de office 365 y 311 la plataforma google classroom tal como se muestra en el gráfico 1. Se puede apreciar que los estudiantes utilizan en gran medida YouTube para aprender mediante videos tutoriales y como herramienta para realizar trabajo colaborativo utilizan la red social Whatsapp.

Figura 1

Herramientas TICS utilizadas por los estudiantes de la FCMF

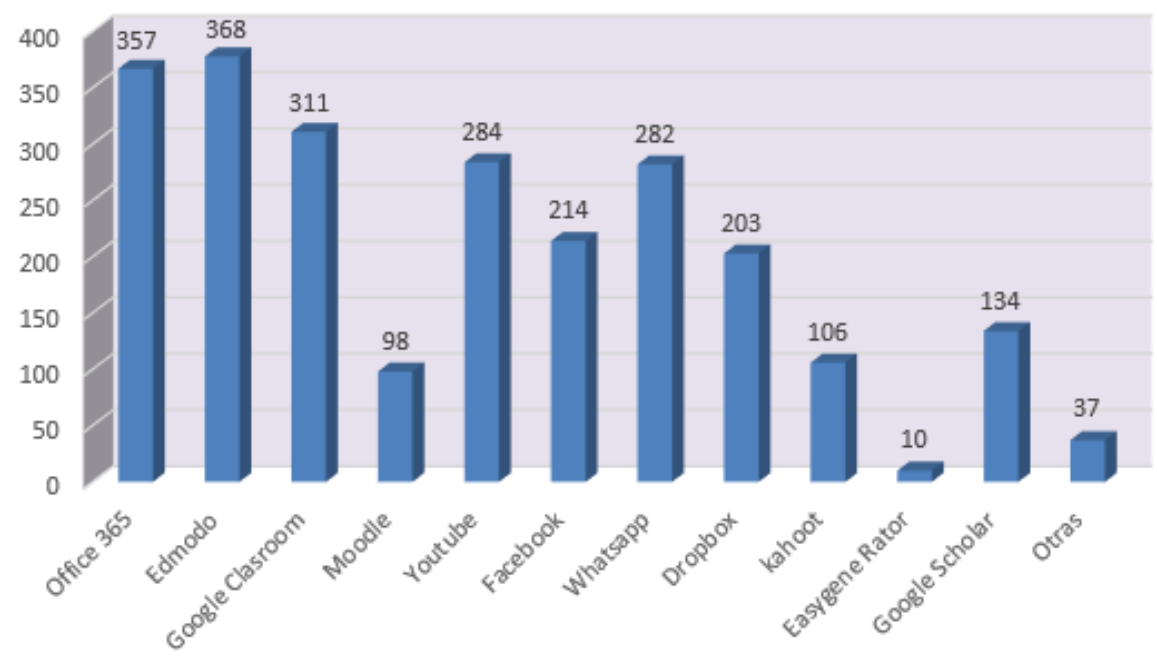


El $84 \%$ de los encuestados indicó que utiliza chats, foros y mensajería instantánea como herramientas de contacto asincrónico con sus docentes, lo cual es importante seguir fomentando para optimizar los procesos de aprendizaje.

Otro resultado obtenido de los estudiantes concluyó con el $84.81 \%$ en que era necesario realizar cambios en los métodos de enseñanza aplicados por los docentes. Era importante conocer esta información para generar cambios e innovar en las metodologías de enseñanza que se apliquen, donde se involucre el uso de las TICS, pues al ser una facultad que ofrece carreras técnicas es importante estar a la vanguardia de los cambios tecnológicos que se presentan constantemente.

El 73.8\% de los encuestados consideraron que si se aplican metodologías apoyadas en el uso de las TICS se contribuirá a reforzar conocimientos y mejorar el rendimiento académico de los estudiantes de la FCMF.

\subsection{Discusión}

Al hacer una visita por los salones de clase de la Facultad se pudo constatar que muchos docentes no usaban TICS, solo se apoyaban en el pizarrón, o realizaban talleres en clase formando con los estudiantes equipos de trabajo los cuales se integraban sin manejar ningún criterio de selección previo, esto se debe a que muchos profesores especialmente de la carrera de Ingeniería Civil fueron formados en otrás épocas y por tanto no están acostumbrados al uso de este tipo de herramientas complementado que la universidad no ha ofrecido ningún tipo de capacitaciones inherentes al uso de estas tecnologías, no se discute el valor de estos métodos tradicionales de aprendizaje pero también es necesario actualmente la incorporación de herramientas relacionadas con las TICS en el proceso de enseñanza, lo cual va de la mano con los adelantos científico-técnicos que existen.

La Universidad de Guayaquil cuenta desde el 9 de junio del 2014 con la plataforma Office 365 de manera gratuita, Microsoft entregó 120000 cuentas para todos los miembros que formaban parte de su comunidad educativa como una contribución para fomentar el uso de las TICS. Este convenio tenía como objetivo beneficiar tanto a profesores como estudiantes motivando su uso para aumentar la productividad, facilitar el trabajo colaborativo y familiarizarse con esta herramienta la cual es ampliamente utilizada en el ámbito laboral. Se promovió un proceso para la capacitación y difusión de la plataforma, pero últimamente no ha existido un plan de capacitación continua para instruir a los estudiantes y profesores que han ingresado a la universidad y esa es una falencia que se debe corregir para potenciar el uso de esa herramienta. Actualmente la tecnología ha ido avanzando de tal forma que se han incorporados los ambientes virtuales de aprendizaje que con ayuda del E-learning, sumado al nuevo paradigma de la computación en la nube y la utilización de la plataforma digital emergente, además del libre acceso al Wi-fi dentro del campus pueden contribuir enormemente al mejoramiento del aprendizaje.

Se debe fomentar el uso de las TICs en asignaturas orientadas especialmente a software y hardware tal como se sugiere en otros estudios similares al descrito, esto permitirá en los estudiantes generar una mayor autonomía y responsabilidad en el proceso de aprendizaje obligando también al docente a implementar estrategias andragógicas innovadoras que cambien los paradigmas de enseñanza aprendizaje tradicionales.

\section{Conclusiones}

Con la información que se obtuvo a partir del análisis realizado en esta investigación se puede concluir que los estudiantes de la Facultad de Ciencias Matemáticas y Físicas de la Universidad de Guayaquil consideran que el uso de las TICS eficientemente en el proceso educativo contribuirá a mejorar su rendimiento académico.

Las TICS (tecnologías de la información y la comunicación), utilizadas como medios de aprendizaje, se complementan a los cambios conductuales de los individuos. El estímulo que tiene el uso de estos recursos 
tecnológicos y herramientas de enseñanza, refuerza el aprendizaje, además de estimular la creatividad, más aún en el área de la informática. Sin embargo, para poder alcanzar esa excelencia educativa se necesita presionar al cambio institucional para de esta manera lograr innovaciones educativas de éxito.

De acuerdo con la información obtenida durante el desarrollo de la investigación a partir del análisis de los datos, es factible concluir que, las prácticas formativas de los estudiantes mediante el uso de tics se ven afectadas por varios factores entre ellos: el nivel socioeconómico que tienen, pues una gran cantidad de alumnos no cuentan con un computador adecuado y la conectividad apropiada en sus hogares que les permita tener una mejor interacción con sus profesores, también la infraestructura que ofrece la facultad no está acorde a la constante evolución que presenta la tecnología actualmente, es primordial reemplazar el hardware y el software instalado en los equipos existente en los laboratorios, se mejorar la conectividad inalámbrica y los espacios de aprendizaje tecnológicos con los que se cuenta y sobre todo los docentes deben generar y despertar en los dicentes desafíos que le permitan afianzar los procesos de aprendizaje mediante estrategias que mantengan su constante interés por descubrir generar aprendizajes significativos.

\subsection{Recomendaciones}

Se recomienda que las autoridades organicen cursos de capacitación y actualización en el uso de las TICS para los estudiantes y especialmente docentes de la Institución donde se realizó el estudio. La formación y actualización permanente constituye siempre una necesidad y una exigencia para mejorar y elevar la calidad del proceso enseñanza aprendizaje en las Instituciones de Educación Superior.

Como facultad de Ciencias Matemáticas se debe propender a generar nuevas ideas y técnicas de aprendizaje modernas usando las tecnologías de información y comunicación de tal forma que se pueda garantizar que los estudiantes desarrollen las habilidades cognitivas necesarias tales como la creatividad y la resolución de problemas que les permita luego alcanzar el éxito en su carreras profesionales.

También es evidente la necesidad de realizar ajustes metodológicos en las asignaturas que están en las diferentes mallas curriculares de las tres carreras de la Facultad, de tal forma que se generen espacios que permitan utilizar las TICS de forma eficiente y así se contribuya en la formación de profesionales que satisfagan las demandas de la sociedad actual.

Se debe fomentar por parte de todos los involucrados el mayor uso de los recursos tecnológicos con los que cuenta la Universidad especialmente los que ofrece la plataforma Office 365 la cual permite usar herramientas tales como One drive, forms, Microsoft teams para manejar aulas virtuales y otros que permitirán tener una mejor interacción entre estudiante y profesor de forma sincrónica y asincrónica mejorando de esta manera el proceso de enseñanza-aprendizaje.

\section{Referencias bibliográficas}

Acosta, J. Z., Vallejo, M., \& Uribe, I. (2017). Estrategias de aprendizaje mediadas tecnológicamente para potenciar el aprendizaje significativo.

Bawden, R. (2009). El propósito educativo de la educación superior para el desarrollo humano y social en el contexto de la globalización. En la educación superior en tiempos de cambio. Nuevas dinámicas para la responsabilidad social. Mundi-Prensa.

Castellano Ascencio M. (2013), Arboleda Montoya BH. Relación estrategias didácticas y TIC en el marco de prácticas pedagógicas de los docentes de instituciones educativas de Medellín. Revista Virtual de la Universidad Católica del Norte [Internet].2013. Disponible en: http://www.redalyc.org/pdf/1942/194225730005.pdf 
Cujia S., Martelo R. Modelo Teórico-Metodológico para la Aplicación de las Tecnologías de Información y comunicación en el Contexto Universitario (2017). Revista Espacios Vol. 38, página 2.

Flórez M., Aguilar A. (2017). Sociedad del conocimiento, las TIC y su influencia en la educación, Revista Espacios. Volumen 38, página 39.

García B., Loredo J., Carranza G. (2008). Análisis de la práctica educativa de los docentes: pensamiento, interacción y reflexión. Revista Electrónica de Investigación Educativa, México; Volumen 10. Disponible en: http://redie.uabc.mx/NumEsp1/contenido-garcialoredocarranza.html

Hernández R. Impacto de las TIC en la educación: retos y perspectivas. Rev. Propósitos y Representaciones [Internet]. 2017 [citado 3 Feb 2018]; 5(1):[aprox. 12 p.]. Disponible en: http://revistas.usil.edu.pe/index.php/pyr/article/view/149/251

Maloney, E. J. (2007). What Web 2.0 can teach us about learning. Chronicle of Higher Education, 53 (18), B26.

Rodríguez Izquierdo, R. (2010). El impacto de las TIC en la transformación de la enseñanza universitaria: Repensar los modelos de enseñanza y aprendizaje. TESI (Teoría de la Educación. Educación y Cultura en la Sociedad de la Información), 11(3), 32-68.

Villalva E. Villalva M. (2018). El aprendizaje de asignaturas del área de Hardware utilizando metodologías andragógicas, Congreso $\mathrm{CISCl} 2018$.

Viñals Blanco A, Cuenca Amigo J. (2015). El rol del docente en la era digital. Revista Interuniversitaria de Formación del Profesorado [Internet]. [aprox. 13 p.]. Disponible en: http://www.redalyc.org/articulo.oa?id=27447325008

Esta obra está bajo una Licencia Creative Commons Attribución-NoCommercial 4.0 International

\section{(cc) EY-NG}

